\title{
Plasmodium berghei ANKA infection induces thymocyte apoptosis and thymocyte depletion in CBA mice
}

\author{
Leonardo JM Carvalho/ ${ }^{+}$, Maria F Ferreira-da-Cruz ${ }^{++}$, Claudio T Daniel-Ribeiro/ ${ }^{++}$, \\ Marcelo Pelajo-Machado*, Henrique L Lenzi*
}

\author{
Laboratório de Pesquisas em Malária, Departamento de Imunologia *Departamento de Patologia, \\ Instituto Oswaldo Cruz-Fiocruz, Av. Brasil 4365, 21045-900 Rio de Janeiro, RJ, Brasil
}

\begin{abstract}
Immune responses to malaria infections are characterized by strong $T$ and $B$ cell activation, which, in addition of potentially causing immunopathology, are of poor efficacy against the infection. It is possible that the thymus is involved in the origin of immunopathological reactions and a target during malaria infections. This work was developed in an attempt to further clarify these points. We studied the sequential changes in the thymus of CBA mice infected with Plasmodium berghei ANKA, a model in which 60-90\% of the infected animals develop cerebral malaria. During the acute phase of infection, different degrees of thymocyte apoptosis were recorded: (1) starry-sky pattern of diffuse apoptosis with maintenance of cortical-medullary structure; (2) intense apoptosis with cortical atrophy, with absence of large cells; (3) severe cortical thymocyte depletion, resulting in cortical-medullary inversion. In the latter, only residual clusters of small thymocytes were observed within the framework of epithelial cells. The intensity of thymus alterations could not be associated with the degree of parasitemia, the expression of clinical signs of cerebral malaria or intensity of brain lesions. The implications of these events for malaria immunity and pathology are discussed.
\end{abstract}

Key words: malaria - thymus - Plasmodium berghei - mice

Malaria infections are characterized by defective immune responses, with poor efficacy and, in some cases, with the occurrence of immunopathology. The major lifethreatening malaria-related complications - cerebral malaria and severe anemia - have a strong immunological component in humans (Mackintosh et al. 2004) as well as in experimental models (De Souza \& Riley 2002, Carvalho et al. 2003).

The Plasmodium berghei ANKA infection in CBA mouse is an established model of malaria with neurological involvement (the so-called experimental cerebral malaria), and infection with blood stage parasites leads to $100 \%$ lethality. The immune response in this model is not only ineffective against parasite growth but also responsible for the $60-90 \%$ incidence of cerebral malaria (CM), mainly characterized by strong Th1 T cell responses (Hunt \& Grau 2003), macrophage hyperactivation (Carvalho et al. 2000) and also $\mathrm{CD}^{+} \mathrm{T}$ cell cytotoxicity (Belnoue et al. 2002). T lymphocytes are central in CM pathogenesis. Early thymectomized CBA mice become resistant to CM development (Grau et al. 1986). Treatment of adult mice with anti-CD4 or anti-CD8 monoclonal antibodies also abrogate $\mathrm{CM}$ development, depending on the moment they are administered - early in infection for anti-CD4 and late in the infection for anti-CD8 (Grau et al. 1986, Belnoue et

${ }^{+}$Corresponding author: leojmc @ ioc.fiocruz.br

${ }^{++}$Recipients of CNPq grants

Received 20 December 2005

Accepted 19 May 2006 al. 2002). CM seems to be mediated by cells bearing $\alpha \beta \mathrm{T}$ cell receptors (TCR), since mice lacking T cells of the $\alpha \beta$ lineage (TCR $\alpha \beta^{-/-}$), but not of the $\gamma \delta$ lineage (TCR $\gamma \delta^{-/-}$), were resistant to CM development (Boubou et al. 1999). In addition, these authors showed that expression of CM was associated with an increase in $\mathrm{T}$ cells bearing the V $\beta 8.1$ and V $\beta 8.2$ segments, and CM expression was reduced in mice rendered deficient in cells bearing these segments.

Although all the events concerning the participation of $\mathrm{T}$ cells in CM physiopathogenesis are related to peripheral responses, events occurring in the thymus cannot be disregarded. Indeed, infection by other protozoan parasites can potentially affect thymic function and then modulate $\mathrm{T}$ cell responses. In Trypanosoma cruzi infection in Balb/c mouse, intense thymic atrophy is reported, with loss of double positive immature thymocytes and appearance of such immature cells in secondary lymphoid organs (Mendes-da-Cruz et al. 2003). In addition, lymphocytes carrying normally forbidden TCR V $\beta$ families are also exported from the thymus. According to these authors such cells could play a role in the autoimmune responses commonly observed during $T$. cruzi infections. Therefore, infection can affect thymic function resulting in pathological consequences or altering the efficacy of the immune response. In malaria, the consequences of malaria infection on thymus structure and function have been overlooked, with few works on the subject (Krettli \& Nussenzweig 1974). In the present work, we evaluated the sequential histological changes taking place in the thymus of CBA mice during $P$. berghei ANKA infection and analyze their possible implications in the protective and/or pathological immune responses in this model of severe malaria. 


\section{MATERIALS AND METHODS}

Animals, parasite and infection - Four to 8 week-old female CBA mice (Cecal-Fiocruz, Rio de Janeiro) were inoculated by intraperitoneal injection with $1 \times 10^{6} \mathrm{P}$. berghei ANKA-parasitized red blood cells obtained from a donor infected CBA mouse. A total of 49 mice in two separate experiments were infected and the thymus of 27 mice were examined by histology, on the following days after infection: 3 (10 mice); 6 (2 mice); 7 (6 mice); 8 ( 2 mice); and 10 (7 mice). Seventeen uninfected control mice (age and sexmatched) were examined on days: 3 (6 mice); 7 (6 mice); and 10 (5 mice). The protocols were reviewed and approved by the Fiocruz Ethical Committee on Animal Experimentation (CEUA protocol number P-0155-03).

Parasitemia and clinical parameters - Density and maturation of circulating parasites were daily determined by examination of Giemsa-stained blood smears collected from the tail vein. Clinical CM was defined by the presentation of at least one of the following signs: limb paralysis, convulsions and roll-over movements.

Histopathologic study - The thymuses were fixed in Carson's formalin-Millonig (Carson et al. 1993) and embedded in paraffin. Sections $(5 \mu \mathrm{m})$ were stained with Hematoxylin-Eosin or Lennert's Giemsa (Lennert 1978) and analyzed by bright-field microscopy.

Statistical analysis - The Mann-Whitney non-parametric test was used to evaluate the differences in the course of parasitemia between the two experiments. The Spearman $r$ non-parametric test was used to examine the correlations between the intensity of thymocyte apoptosis and the relevant events analyzed (parasitemia, intensity of brain alterations, and expression of clinical signs of $\mathrm{CM})$. P values lower than 0.05 were considered significant.

\section{RESULTS}

The two experiments provided similar results. Although the mean parasitemia was higher in the mice of the experiment 2 in the first 4 days, and the opposite situation was observed from day 8 onwards (Fig. 1), the differences in the course of parasitemia between the two experiments were not significant. In addition, in the experiment 2 there was an earlier mortality due to cerebral malaria and a higher incidence of this complication (Fig. 2), but again these differences were not significant.

Ten out of the 49 infected mice were killed on day 3 of infection, their organs collected and examined to check for early histopathological changes. Among the remaining 39 mice, 23 (59\%) displayed clinical signs of CM on days 5-8 of infection. During this period, 6 mice with clinical signs of CM were killed (two mice in each day: 6, 7 and 8 ) and their organs collected. In addition, 6 other mice without clinical signs of CM were killed on day 7 and their organs collected. All the 12 animals killed on days 6-8, with or without clinical signs of CM, had histopathological evidence of brain lesions, varying from mild (few microhaemorrhagic foci and/or incipient monocyte adhesiveness to endothelium) to intense (diffuse haemorrhagic foci, intense vascular plugging by pigment containing-

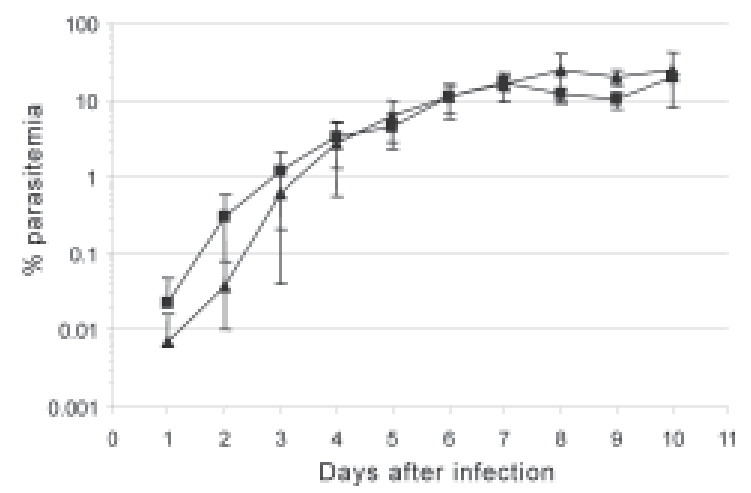

Fig. 1: course of parasitemia (mean \pm standard deviation) of Plasmodium berghei ANKA in CBA mice inoculated with $1 \times 10^{6}$ parasitized red blood cells; triangles: experiment 1; squares: experiment 2 .

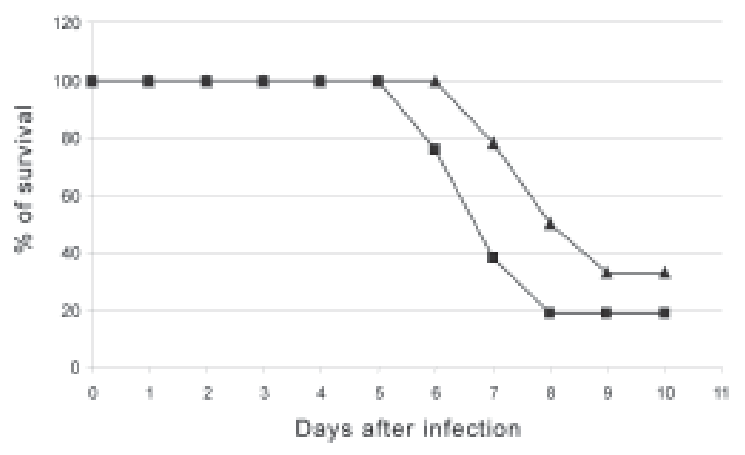

Fig. 2: survival curve of CBA mice infected with $1 \times 10^{6}$ Plasmodium berghei ANKA-parasitized red blood cells; triangles: experiment 1; squares: experiment 2 .

monocytes, perivascular edema). The 10 mice that remained did not develop clinical CM and were killed on day 10 . One of these 10 mice, which presented a delayed course of parasitemia, was the only presenting histological alterations in the brain associated with CM (haemorrhagic foci, vascular plugging by pigment containing-monocytes, perivascular edema). Detailed description of the lesions in the brain, liver, and lungs has been published (Carvalho et al. 2000).

Histopathology of the thymus - Thymuses of noninfected control mice showed thymocytes of variable size (small, medium, and large) in the cortical region, distributed in a typical organization in cell clusters (Fig. 3A). Mitotic cells were observed and apoptosis was minimal and speckled. Thymuses of infected mice on day 3 were similar to controls (Fig. 3B); the only changes were the presence of a few malarial pigment-containing monocytes in blood vessels and a few foci of thymocyte apoptosis (Fig. 3C). The thymuses of all mice killed on days 6-8 of infection presented lymphocytic apoptosis in the cortical region. The intensity of apoptosis was variable among the 10 mice analyzed and could be divided in three patterns: (1) a starry-sky pattern of diffuse apoptosis with maintenance of cortical-medullary structure (3 mice - Fig. 3D-E); (2) intense apoptosis with cortical atrophy (4 mice), 
with absence of large cells - only small thymocytes could be seen, without mitotic figures; (3) severe cortical thymocyte depletion, resulting in cortical-medullary inversion (3 mice); only residual clusters of small thymocytes were observed within the framework of epithelial cells (Fig.
$3 \mathrm{~F})$. These thymuses were very small compared to normal controls, both at the macroscopic and microscopic levels, denoting intense thymic atrophy. On day 10, thymuses of 7 out of the 10 mice killed were analyzed. Three of 7 mice analyzed presented thymus with a well-conserved struc-
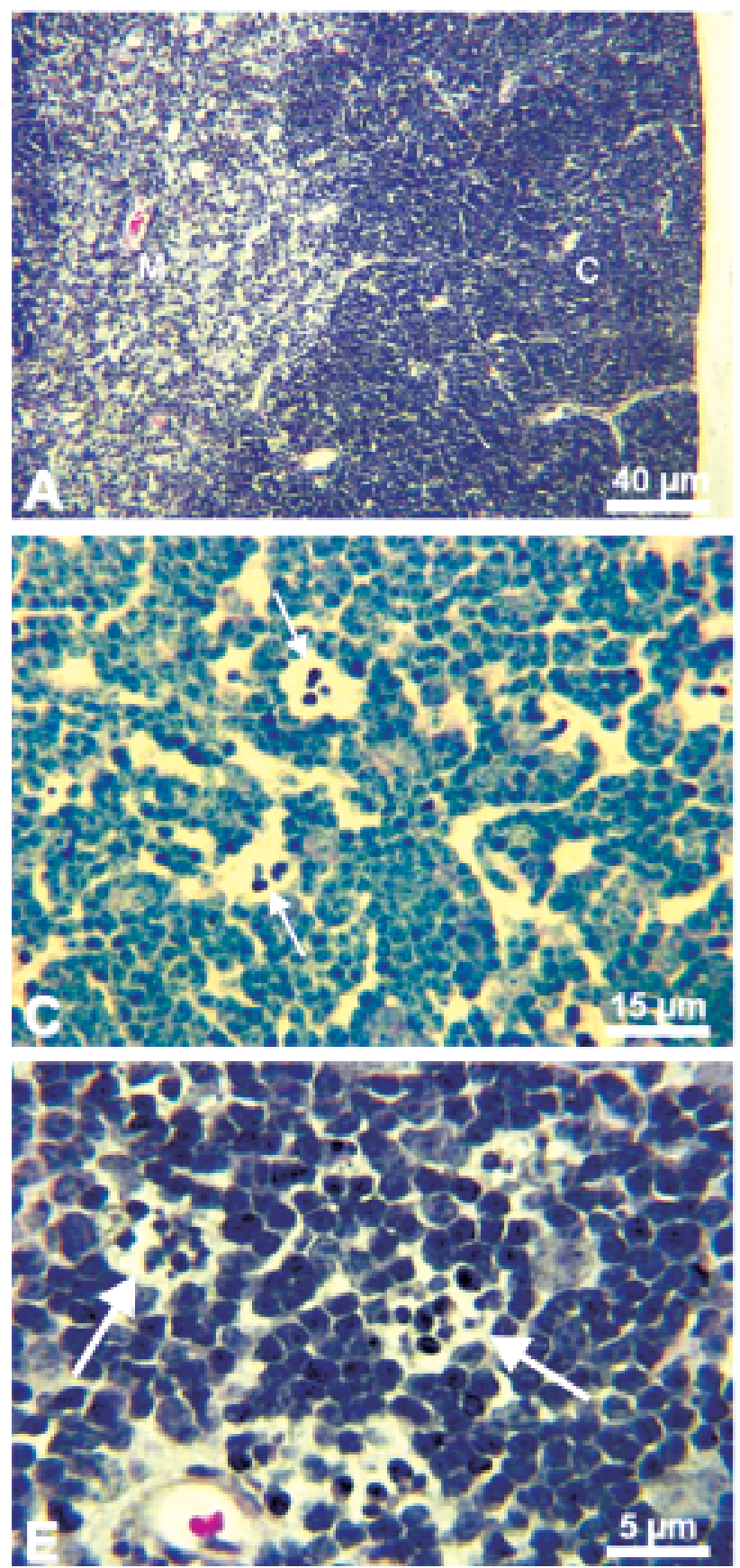
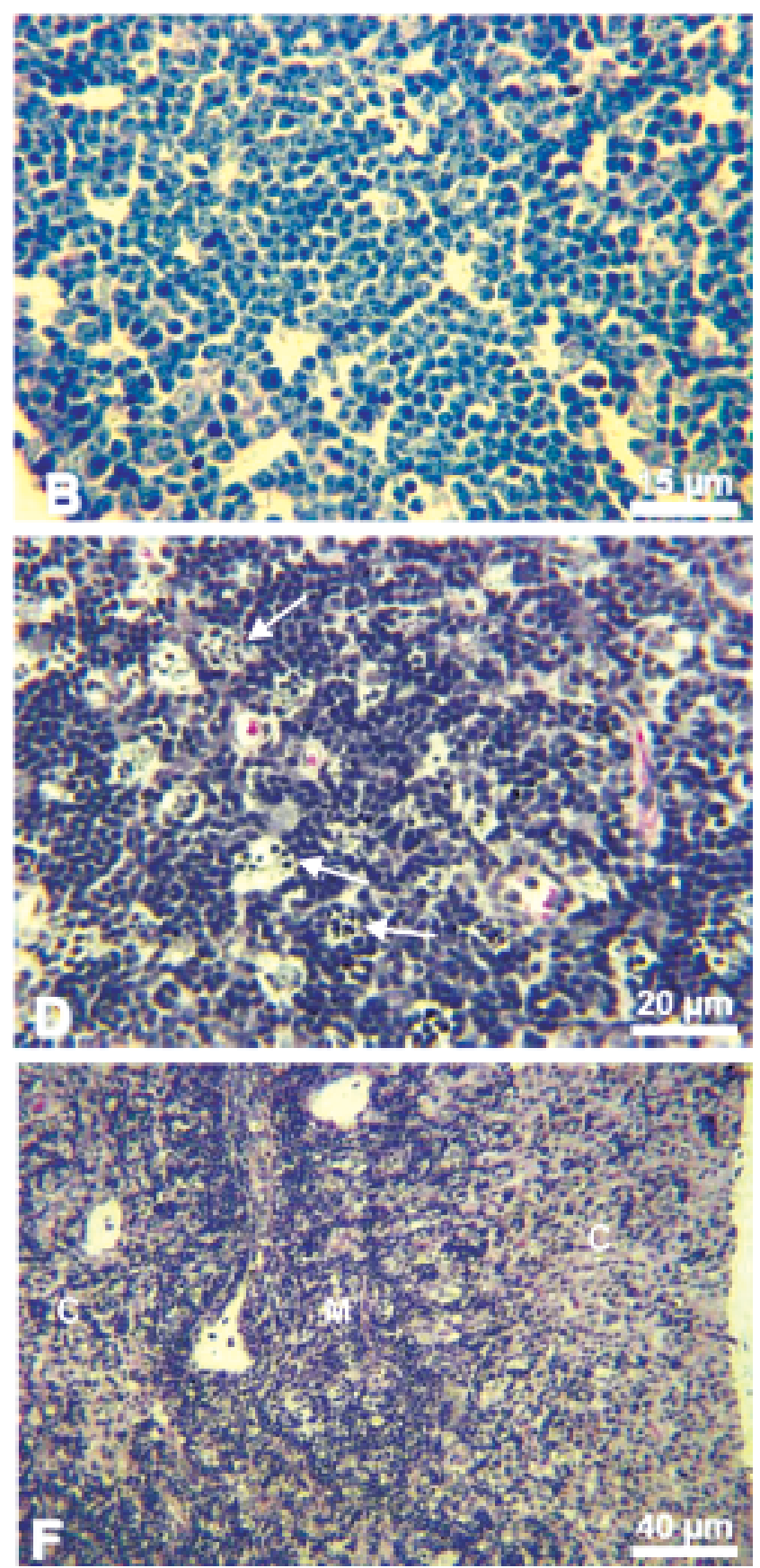

Fig. 3A: panoramic view of a normal mouse thymus, showing the cortical area with plenty of thymocytes and the internal medullar area with a lower cellularity; B: thymic cortical area of a Plasmodium berghei ANKA-infected mouse (day 3 ). The aspect is very similar to noninfected controls; C: thymic cortical area of a P. berghei ANKA-infected mouse (day 3), showing initial foci of apoptotic thymocytes; D: thymic cortical area of a P. berghei ANKA-infected mouse (day 7), showing a starry-sky pattern of widespread thymocyte apoptosis, and the persistence of medium-sized thymocytes; E: thymic cortical area of a $P$. berghei ANKA-infected mouse (day 7), showing the foci of thymocyte apoptosis in higher detail, and the persistence of medium-sized thymocytes; F: panoramic view of the thymus of a $P$. berghei ANKA-infected mouse (day 8), showing intense thymocyte depletion in the cortical area, with only a few remaining thymocytes in between the framework of epithelial cells, and an increase in the medullar cellularity resulting in cortico-medullar inversion. Arrows indicate some of the apoptosis foci (Figs 3C, D, and E); C: cortex; M: medulla. In the Figs 3A, D, E, and F the thymuses were stained with Hematoxilin-eosin, and in Figs $3 \mathrm{~B}$ and $\mathrm{C}$ the thymuses were stained with Giemsa. 
ture; one mouse had cortical atrophy but with persistent medium-sized thymocytes; and 3 of them showed strong cortical atrophy with cortical-medullary inversion. The latter also presented prominent Hassal's bodies, sometimes cystified. Plasma cells, eosinophils and myelopoiesis foci were observed in some of the mice, and mast cells apparently increased in number. One of the mice displayed many immunoblasts in the cortical region. Thymuses of 5 mice (two on day 7 and three on day 10) are not shown because they presented strong atrophy and were so small that no good sections were obtained.

The Table shows a summary of the data for each infected mouse. No correlation could be determined between intensity of thymus apoptosis and either parasitemia, expression of clinical signs of CM or degree of brain alterations. For instance, the mildest (starry-sky pattern of apoptosis) or the most severe (cortico-medullary inversion) thymic alterations were found in mice with or without clinical signs of CM. Accordingly, mice presenting either mild or intense brain lesions eventually developed thymic cortico-medullary inversion.

\section{DISCUSSION}

We show that $P$. berghei ANKA infection in CBA mice can lead to striking changes in the thymus, with intense thymocyte apoptosis, cell depletion and organ atrophy. Thymus atrophy can be induced by other infectious agents, such as rabies virus (Marcovistz et al. 1994), $T$. cruzi (Savino et al. 1989) and AIDS in animal models, as well as in malignant diseases (Tshikuka et al. 1992). In acute $T$. cruzi infection in mice, severe thymic atrophy is mainly due to depletion of $\mathrm{CD} 4^{+} \mathrm{CD}^{+}$double positive thymocytes by apoptosis (Leite-de-Moraes et al. 1992). Preliminary results in our laboratory shows that thymocyte depletion in CBA mice during $P$. berghei ANKA infection is also mainly due to great decrease in $\mathrm{CD}^{+} \mathrm{CD}^{+}$double positive cells, also observed in Balb/c mice (unpublished observation). The occurrence of thymic atrophy in the CM-resistant Balb/c mouse strain suggests that thymic microenvironment changes are not directly linked to $\mathrm{CM}$ pathogenesis. This interpretation is strengthened by the lack of relationship, in the present work, between intensity of thymic alterations and other parameters, such as parasitemia, expression of clinical signs of CM and intensity of brain lesions, suggesting the independence of these phenomena. However, since there was a range of variation in each of the parameters analyzed, this conclusion may not be definitive, and experiments with larger groups of animals will be necessary to confirm these data.

TABLE

Parasitemia, expression of clinical signs of cerebral malaria, and intensity of histological alterations in the thymus and brain of CBA mice after 6-10 days of infection with Plasmodium berghei ANKA

\begin{tabular}{|c|c|c|c|c|c|}
\hline $\begin{array}{l}\text { Mouse code } \\
\text { (Experiment) }\end{array}$ & $\begin{array}{l}\text { Day of } \\
\text { sacrifice }\end{array}$ & $\begin{array}{c}\text { Parasitemia } \\
\text { (in the day of sacrifice) }\end{array}$ & $\begin{array}{l}\text { Thymus histology } \\
\text { (intensity of alterations) }\end{array}$ & $\begin{array}{c}\text { Expression of } \\
\text { clinical CM }\end{array}$ & $\begin{array}{c}\text { Brain histology } \\
\text { (intensity of alterations) }\end{array}$ \\
\hline $8464(\operatorname{Exp} 2)$ & 6 & 19.8 & + & Yes & ++++ \\
\hline $8465(\operatorname{Exp} 2)$ & 6 & 16.3 & +++ & Yes & +++ \\
\hline $8466(\operatorname{Exp} 2)$ & 7 & 21.0 & ND & Yes & ++++ \\
\hline $8467(\operatorname{Exp} 2)$ & 7 & 15.2 & +++ & No & +++ \\
\hline $8468(\operatorname{Exp} 2)$ & 7 & 15.5 & ++ & No & ++ \\
\hline $8487(\operatorname{Exp} 2)$ & 7 & 11.1 & +++ & No & +++ \\
\hline $8295(\operatorname{Exp} 1)$ & 7 & 20.4 & + & No & + \\
\hline $8296(\operatorname{Exp} 1)$ & 7 & 12.2 & + & No & + \\
\hline $8297(\operatorname{Exp} 1)$ & 7 & 14.0 & ND & Yes & ++ \\
\hline $8298(\operatorname{Exp} 1)$ & 7 & 19.8 & ++ & No & + \\
\hline $8498(\operatorname{Exp} 1)$ & 8 & 33.3 & +++ & Yes & +++ \\
\hline $8499(\operatorname{Exp} 1)$ & 8 & 24.7 & ++ & Yes & +++ \\
\hline $8491(\operatorname{Exp} 2)$ & 10 & 21.6 & 0 & No & $+/-$ \\
\hline $8492(\operatorname{Exp} 2)$ & 10 & 17.0 & +++ & No & $+1-$ \\
\hline $8493(\operatorname{Exp} 2)$ & 10 & 19.6 & ++ & No & $+1-$ \\
\hline $8494(\operatorname{Exp} 2)$ & 10 & 20.1 & +++ & No & $+1-$ \\
\hline $8303(\operatorname{Exp} 1)$ & 10 & 11.4 & ND & No & ++ \\
\hline $8304(\operatorname{Exp} 1)$ & 10 & 41.1 & +++ & No & $+/-$ \\
\hline $8305(\operatorname{Exp} 1)$ & 10 & 34.9 & ND & No & 0 \\
\hline $8306(\operatorname{Exp} 1)$ & 10 & 16.0 & 0 & No & $+1-$ \\
\hline $8307(\operatorname{Exp} 1)$ & 10 & 14.1 & ND & No & 0 \\
\hline $8308(\operatorname{Exp} 1)$ & 10 & 29.6 & 0 & No & 0 \\
\hline
\end{tabular}

Intensity of thymic alterations - 0 : similar to normal controls; +: starry-sky pattern of apoptosis; ++: intense apoptosis, with persistence of small thymocytes; +++: cortical thymocyte depletion, with cortico-medullary inversion. Intensity of brain alterations - 0: similar to normal controls; +/-: mild vessel plugging by pigment-containing monocytes with or without mild perivascular edema and without hemorrhages; + few microhemorrhages with or without mild monocyte adherence and vessel plugging; ++: hemorrhages with more intense vessel plugging by pigment-containing monocytes, perivascular edema; +++: diffuse hemorrhages, intense adherence of heavily pigment-loaded monocytes leading to occlusion of parenchyma vessels and formation of "cell nets" inside large subarachnoid vessels, perivascular edema with leukocyte extravasation; ++++: same as +++ plus huge subarachnoid hemorrhages. ND: not determined. 
Although apparently not relevant to brain pathology, thymic atrophy with thymocyte depletion may have important implications for the development of immunity to malaria blood stage infection. Disturbance of the thymus microenvironment may lead to changes in the peripheral $\mathrm{T}$ cell repertoire, and may also lead to the release of $T$ cells having a role in the response to autoantigens, as commonly observed during malarial infections (Daniel-Ribeiro \& Zanini 2000). In normal thymuses, autoimmune T cell clones emerging during maturation of thymocytes are deleted through the mechanism of negative selection. It is possible that, in thymuses presenting changes in the microenvironment, autoimmune clones might skip such mechanisms. This phenomenon seems to happen, for instance, in the T. cruzi infection of mice. In this case, the number of $\mathrm{CD}^{+}{ }^{+} \mathrm{CD} 8^{+}$double positive cells, which usually represents immature $\mathrm{T}$ cells normally found only in the thymus, greatly increases in lymph nodes. In addition, among the double positive as well as the single positive $\mathrm{CD}^{+}{ }^{+}$or $\mathrm{CD}^{+}$cells, there is a significant increase of cells bearing prohibited $\mathrm{V} \beta \mathrm{T}$ cell receptors such as $\mathrm{V} \beta 5, \mathrm{~V} \beta 8$, and $V \beta 12$, normally not expressed in Balb/c mice (Mendesda-Cruz et al. 2003). Selective increase in blood T cells carrying the TCR V $\beta 8$ has been described in P. berghei ANKA clone 1.49L infection in the B10.D2 mouse strain (Boubou et al. 1999), and the authors suggest that the selective TCR Vb8 expansion might be implicated in CM pathogenesis. Although this increase was due to peripheral clone expansion and the thymus was not analyzed in that work, changes in the $\mathrm{T}$ cell repertoire and deficiency in $\mathrm{T}$ cell maturation due to infection-mediated thymus damage are possible mechanisms interfering in malaria immunity and deserve further investigations.

Development of an effective malaria vaccine has been so far a disappointing enterprise, and one of the main reasons for the failure is the limited knowledge on the mechanisms of immunity (Carvalho et al. 2002). Therefore, understanding the implications of thymus atrophy and thymocyte depletion on the acquisition of immunity to malaria is an important issue. In this sense, it would be interesting to verify what happens to the thymus after recovery from infection in a non-lethal model, as well as the thymus alterations during chronic infection, since this may be a contributor to the poor efficacy of immune responses to malaria infections in areas of high malaria transmission, where individuals are continuously exposed to the parasite. The data presented here then open perspectives for studies to demonstrate if and how thymus damage can influence immunity and pathology during malaria infections.

\section{ACKNOWLEDGEMENTS}

To Belmira Santos from the Fiocruz Animal House for providing the CBA mice, and Bruno Eschenazi from the Laboratory of Production and Treatment of Images/IOC/Fiocruz for processing the images of the thymuses.

\section{REFERENCES}

Belnoue E, Kayibanda M, Vigario AM, Deschemin JC, van Rooijen N, Viguier M, Snounou G, Renia L 2002. On the pathogenic role of brain-sequestered alphabeta $\mathrm{CD} 8^{+} \mathrm{T}$ cells in experimental cerebral malaria. J Immunol 169: 6369-6375.

Boubou MI, Collette A, Voegtle D, Mazier D, Cazenave PA, Pied S 1999. T cell response in malaria pathogenesis: selective increase in T cells carrying the TCR V(beta) 8 during experimental cerebral malaria. Int Immunol 11: 1553-1562.

Carson FL, Martin JH, Lynn JA 1993. Formalin fixation for electron microscopy: a re-evaluation. Am J Clin Pathol 59: 365-373.

Carvalho LJM, Alves FA, Oliveira SG, Valle RR, Fernandes AAM, Muniz JAPC, Daniel-Ribeiro CT 2003. Severe anemia affects both splenectomized and non-splenectomized Plasmodium falciparum-infected Aotus infulatus monkeys. Mem Inst Oswaldo Cruz 98: 679-686.

Carvalho LJM, Daniel-Ribeiro CT, Goto H 2002. Malaria vaccine: candidate antigens, mechanisms, constraints and prospects. Scand J Immunol 56: 327-343.

Carvalho LJM, Lenzi HL, Pelajo-Machado M, Oliveira DN, Daniel-Ribeiro CT, Ferreira-da-Cruz MF 2000. Plasmodium berghei: cerebral malaria in CBA mice is not clearly related to plasma TNF levels or intensity of histopathological changes. Exp Parasitol 95: 1-7.

Daniel-Ribeiro CT 2000. Is there a role for autoimmunity in immune protection against malaria? Mem Inst Oswaldo Cruz 95: 199-207.

Daniel-Ribeiro CT, Zanini GM 2000. Autoimmunity and malaria: what are they doing together? Acta Trop 76: 205-221.

De Souza JB, Riley EM 2002. Cerebral malaria: the contribution of studies in animal models to our understanding of immunopathogenesis. Microbes Infect 4: 291-300.

Grau GE, Piguet PF, Engers HD, Louis JA, Vassali P, Lambert PA 1986. $\mathrm{L} 3 \mathrm{~T}^{+}{ }^{+} \mathrm{T}$ lymphocytes play a major role in the pathogenesis of murine cerebral malaria. J Immunol 137: 2348-2354

Hunt NH, Grau GE 2003. Cytokines: accelerators and brakes in the pathogenesis of cerebral malaria. Trends Immunol 24: 491-499.

Krettli AU, Nussenzweig R 1974. Depletion of T and B lymphocytes during malaria infections. Cell Immunol 13: 440446.

Leite-de-Moraes MC, Honteyberie-Joskowicz M, Dardenne M, Savino W 1992. Modulation of thymocyte subsets during acute and chronic phases of experimental Trypanosoma cruzi infection. Immunology 77: 95-98.

Lennert K 1978. Malignant Lymphoma other than Hodgkin's Disease, Springer-Verlag, Berlin, 833 pp.

Mackintosh CL, Beeson JG, Marsh K 2004. Clinical features and pathogenesis of severe malaria. Trends Parasitol 20: 597-603.

Marcovistz R, Bertho AL, Matos DC 1994. Relationship between apoptosis and thymocyte depletion in rabies-infected mice. Braz J Med Biol Res 27: 1599-1603.

Mendes-da-Cruz DA, de Meis J, Cotta-de-Almeida V, Savino W 2003. Experimental Trypanosoma cruzi infection alters the shaping of the central and peripheral T-cell repertoire. Microbes Infect 5: 825-832.

Savino W, Leite-de-Moraes MC, Honteyberie-Joskowicz M, Dardenne M 1989. Studies on the thymus in Chagas' dis- 
ease. I. Changes in the thymic microenvironment in mice acutely infected with Trypanosoma cruzi. Eur J Immunol 19: 1727-1733.

Tshikuka JG, Torres-Anjel MJ, Blenden DC, Elliot SC 1992.
The microepidemiology of wasting syndrome, a common link to diarrheal disease, cancer, rabies, animal models of AIDS, and HIV-AIDS YHAIDS). The feline leukemia virus and rabies virus models. Ann N Y Acad Sci 653: 274-296. 